\title{
Avaliação ambiental de atividade de dragagem através da aplicação de indicadores de qualidade da água
}

\section{Vinicius de Oliveira Chagas ${ }^{1}$, Sérgio Luiz Costa Bonecker ${ }^{2}$, Mariana Brasil da Cruz ${ }^{1}$}

\begin{abstract}
${ }^{1}$ Universidade Federal do Rio de Janeiro. Escola Politécnica. Av. Athos da Silveira Ramos, 149, CT. Bloco A. 2 andar. Cidade Universitária. Rio de Janeiro-RJ, Brasil (CEP 21941-909).E-mail: vinicius_chagas@poli.ufrj.br.

${ }^{2}$ Universidade Federal do Rio de Janeiro. Instituto de Biologia. Centro de Ciências da Saúde. Departamento de Zoologia. Cidade Universitária. Rio de Janeiro-RJ, Brasil (CEP 21941-902).
\end{abstract}

Resumo. Os conflitos decorrentes da operação de dragagem em relação à qualidade dos corpos hídricos ocorrem, principalmente, em função da ressuspensão dos sedimentos dragados. Para estabelecer o atendimento aos requisitos ambientais da operação de dragagem, neste estudo foi avaliada a qualidade ambiental da água na região da dragagem do Canal do Fundão, localizados Baía de Guanabara, Município do Rio de Janeiro. Essa avaliação foi realizada aplicando a Directiva-Quadro da Água da União Europeia (DQA) e usando como parâmetro a Resolução CONAMA no 357/2005, considerando que a Baía de Guanabara enquadra-se na Classe 1 - Águas Salobras. Foram monitorados as varáveis $\mathrm{pH}$, oxigênio dissolvido, turbidez, amônia, nitrito e nitrato em quatro estações de amostragens. As quatro estações foram posicionadas em torno da draga a um raio de $400 \mathrm{~m}$ na proa, boreste, popa e bombordo da embarcação de dragagem. Para estabelecer o critério de enquadramento com a diretiva foi adotado o limite da referida Resolução CONAMA como de Qualidade Ecológica em "Estado Bom". O oxigênio dissolvido, amônia e turbidez obtiveram resultados fora dos limites considerados. Foi observada a necessidade de ações, de acordo com a DQA, principalmente na região mais próxima a atividade de dragagem.

Palavras-chaves: Dragagem; Qualidade da água; Diretiva-Quadro da Água (DQA).

Abstract. Environmental evaluation of dragging activity through the application of water quality indicators. The conflicts arising from the dredging operation in relation to the quality of the water bodies occur mainly due to the re-suspension of the dredged sediments. In order to establish compliance with the environmental requirements of the dredging operation, this study evaluated the environmental quality of the water in the dredging region of the Canal do Fundão, located at Guanabara Bay, Rio de Janeiro. This evaluation was carried out by applying
Recebido:

12/07/2018

Aceito:

29/08/2018

Publicado:

$31 / 08 / 2018$

Acesso aberto

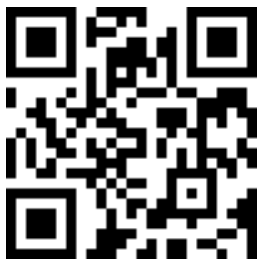

0000-0002-2406-5066

Vinicius de Oliveira Chagas

(D) 0000-0002-4257-7815

Sérgio Luiz Costa

Bonecker

(D) 0000-0001-8872-6989

Mariana Brasil da Cruz 
the EU Water Framework Directive (WFD) and using as reference values the CONAMA Resolution 357/2005, considering that the Guanabara Bay falls within Class 1 - Brackish Water. The variables $\mathrm{pH}$, dissolved oxygen, turbidity, ammonia, nitrite and nitrate were monitored at four sampling stations. The four stations were positioned around the dredge within a radius of $400 \mathrm{~m}$ at the bow, starboard, stern and port of the dredging vessel. In order to establish the criterion of compliance with the Directive, the limit of the CONAMA Resolution as Ecological Quality in "Good state" was adopted. The dissolved oxygen, ammonia and turbidity obtained results outside the limits considered. The need for action was observed, according to the WFD, mainly in the region closest to the dredging activity.

Keywords: Dredging, Water quality and Water Framework Directive (WFD).

\section{Introdução}

De acordo com Cunha (2006) as atividades portuárias e o incremento do comércio exterior ratificam a necessidade de investimentos em obras nas vias de acesso, dragagens, projetos de expansão das instalações que esbarram na falta de regularidade ambiental. Devido a implicações diretamente relacionadas ao meio ambiente, a dragagem é um processo que deve ser regulamentado. Em uma escala global, a Convenção de Londres, realizada em 1972 (CL-1972), identificou que diversas porções marítimas haviam se tornando intensamente degradadas, e que parte dessa degradação havia se dado pela disposição irregular do material dragado. Desta forma, é fundamental que os impactos da atividade sejam mitigados.

Os conflitos decorrentes da operação de dragagem em relação à qualidade dos corpos hídricos ocorrem, principalmente, em função da ressuspensão dos sedimentos dragados, que tem como consequência uma piora na qualidade ambiental da área dragada. Assim, a concordância com os requisitos ambientais apresenta-se como premissa fundamental para viabilidade ambiental das atividades de dragagem e, consequentemente, das atividades portuárias.
Para determinar a qualidade da água, tem-se como instrumento a Resolução CONAMA no 375/2005 (Brasil, 2005), e para avaliar se um determinado corpo d'água apresenta condições satisfatórias para assegurar os seus usos potenciais é necessário efetuar a caracterização física, química e bacteriológica da água.

A Directiva-Quadro da Água no 2000/60/CE (Comunidades Europeias, 2000), por sua vez, é um instrumento integrador da política de recursos hídricos da União Europeia (UE), conhecido por estabelecer um quadro de ação comunitária no domínio da política da água e definir um enquadramento para a proteção e melhoria das águas, prevenção e redução dos níveis de poluição.

Para estabelecer o atendimento aos requisitos ambientais da operação de dragagem, neste estudo será avaliada a qualidade ambiental da água na região da dragagem do Canal do Fundão. Essa avaliação será realizada considerando os parâmetros obtidos no monitoramento realizado na área comparando com os valores de referência estabelecidos pela Resolução CONAMA no 357/2005. Por fim, as águas da baía de Guanabara serão classificadas segundo a diretiva-quadro da água da União Europeia (DQA), para apontar a necessidade ou não da 
execução de medidas mitigadoras (necessidade de ações).

\section{Materiais e métodos}

Para o presente estudo foram consideradas as atividades de um projeto de dragagem realizado no Canal do Fundão, localizados Baía de Guanabara, Município do Rio de Janeiro.

\section{Coleta dos dados}

As estações de amostragens foram localizadas ao redor do centro da atividade de dragagem. Foram posicio- nadas quatro estações concêntricas em torno da draga a um raio de $400 \mathrm{~m}$ na proa, boreste, popa e bombordo da embarcação de dragagem e assim nomeadas:

Ponto de Monitoramento 1 - PM1

Ponto de Monitoramento 2 - PM2

Ponto de Monitoramento 3 - PM3

Ponto de Monitoramento 4 - PM4

A localização das estações variou de acordo com o posicionamento da draga em operação (Figura 1).

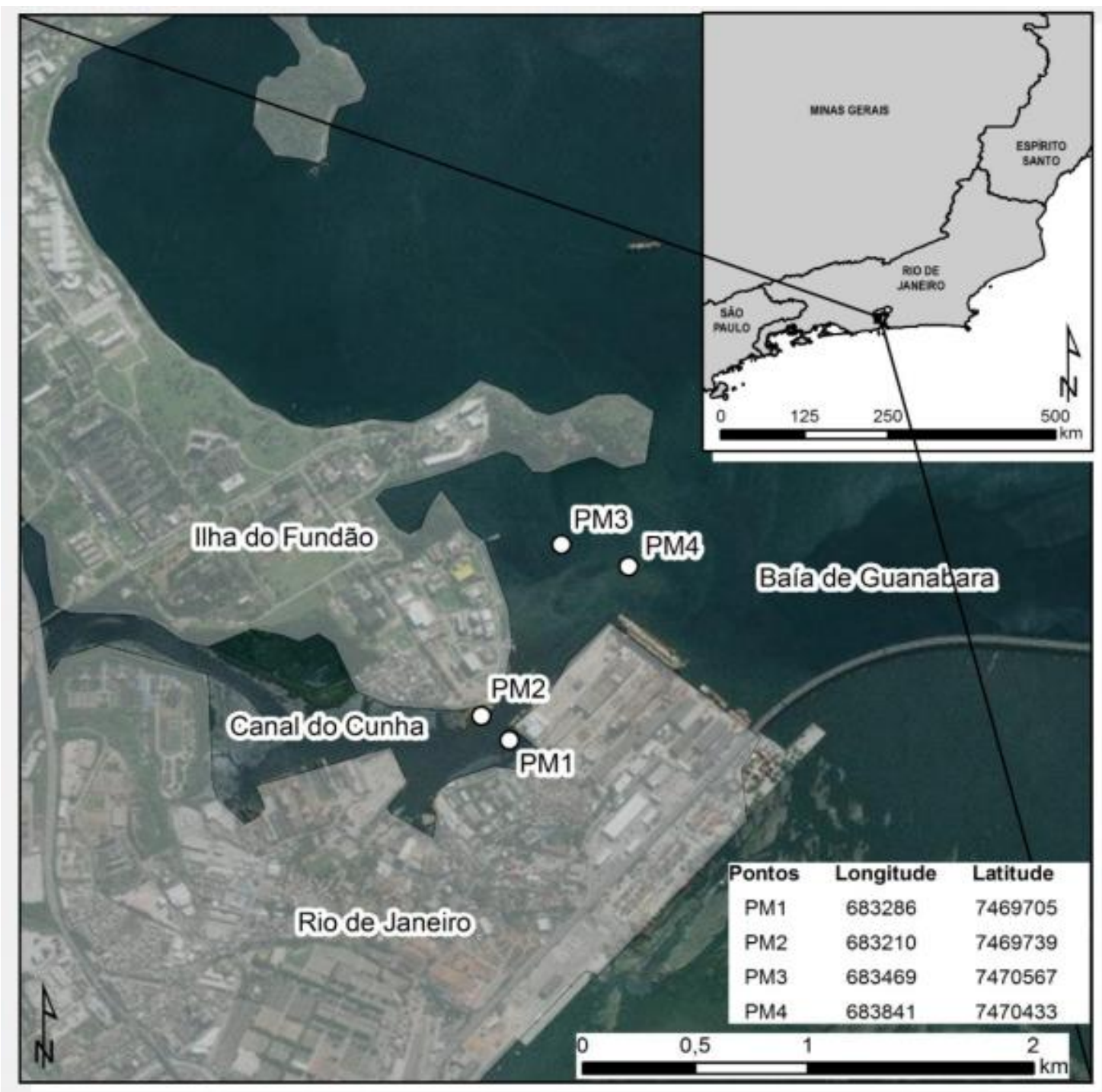

Figura 1. Representação geral da localização dos quatro estações de monitoramento na Baía de Guanabara. 
As amostragens foram realizadas entre dezembro de 2014 e outubro de 2015, em situação de maré enchente e ao longo do monitoramento na área de dragagem, seis dragas, em momentos diferentes, estiveram em operação.

As variáveis físicas e químicas analisadas foram $\mathrm{pH}$, oxigênio dissolvido (OD), turbidez, nitrito, nitrato e amônia. Em todas as estações, as medições da sonda e as coletas de água com garrafa de Niskin foram realizadas tanto na camada superficial (aproximadamente 0,5 m) quanto na de fundo (aproximadamente $0,5 \mathrm{~m}$ acima do fundo). Ressalta-se que as coletas com sonda foram realizadas semanalmente e com a garrafa quinzenalmente.

\section{Avaliação e análise dos dados de acordo com DQA e Resolução CONAMA no 375/2005}

Para determinar a qualidade da água da área de dragagem, foi realizado um paralelo entre a DQA e os parâmetros fornecidos pela Resolução CONAMA $\mathrm{n}^{\mathrm{o}}$ $375 / 2005$, considerando que a Baía de Guanabara enquadra-se na Classe 1 Águas Salobras. Para estabelecer o critério de enquadramento com a diretiva foi adotado o limite da referida resolução como de Qualidade Ecológica em "Estado Bom" (Tabela 1).

Tabela 1. Classificação DQA.

\begin{tabular}{|l|l|l|l|}
\hline \multicolumn{1}{|c|}{ Classe } & Qualidade Ecológica & \multicolumn{1}{c|}{ Cor } & \multicolumn{1}{c|}{ Significado } \\
\hline I & Estado Excelente & & Estado de Referência \\
\hline II & Estado Bom* & & Estado Desejado \\
\hline III & Estado Razoável & & Necessita de Ações \\
\hline IV & Estado Insatisfatório & & Necessita de Ações \\
\hline V & Estado Ruim & & Necessita de Ações \\
\hline
\end{tabular}

*Nota: 0 valor de referência foi baseado nos parâmetros fornecidos pela Resolução CONAMA no 375/2005.

Desta forma, considerando a Resolução CONAMA no 375/2005, foram selecionados os seguintes parâmetros a serem submetidos a DQA: OD, $\mathrm{pH}$, Amônia, Nitrito, Nitrato. Ressalta-se que a referida resolução não estabelece limite para o parâmetro turbidez para Águas Salobras Classe 1 e, dessa forma, foi adotado o padrão de potabilidade da Organização Mundial da Saúde (OMS), conforme Tabela 2.

Tabela 2. Parâmetros estabelecidos pela Resolução CONAMA no 357/2005 e pela Organização Mundial da Saúde.

\begin{tabular}{|c|c|c|}
\hline Parâmetros & Limites & Referência \\
\hline OD & não inferior a $5 \mathrm{mg} / \mathrm{L} \mathrm{O}_{2}$; & \multirow{5}{*}{ Resolução CONAMA no 357/2005 } \\
\hline $\mathrm{pH}$ & entre 6,5 e 8,5 & \\
\hline Amônia & $0,40 \mathrm{mg} \cdot \mathrm{L}^{-1}$ & \\
\hline Nitrito & $0,07 \mathrm{mg} \cdot \mathrm{L}^{-1}$ & \\
\hline Nitrato & $0,40 \mathrm{mg} \cdot \mathrm{L}^{-1}$ & \\
\hline Turbidez & 5 Unidades Nefelométrica de Turbidez (UNT) & $\begin{array}{l}\text { Organização Mundial da Saúde } \\
\text { (OMS). }\end{array}$ \\
\hline
\end{tabular}


A DQA classifica a qualidade ecológica dos corpos d'água em cinco níveis que vão de excelente a ruim, sendo excelente quando há nenhuma ou pouca alteração antropogênica (normalmente associado a condições não perturbadas) e ruim quando os valores dos elementos de qualidade apresentam alterações graves.
Desta forma, e como já explicitado para estabelecer a relação entre a Resolução CONAMA e a DQA, utilizou-se como referência para classificação "boa" os parâmetros já determinados pela resolução. A partir daí foram definidos os parâmetros para cada uma das outras classificações (Tabela 3 e 4).

Tabela 3. Variáveis analisadas: oxigênio dissolvido, pH e turbidez.

\begin{tabular}{|c|c|c|c|}
\hline \multirow{2}{*}{$\begin{array}{c}\text { Qualidade } \\
\text { Ecológica }\end{array}$} & $\begin{array}{c}\text { Oxigênio } \\
\text { dissolvido (mg/L) }\end{array}$ & pH & $\begin{array}{c}\text { Turbidez } \\
\text { (UNT) }\end{array}$ \\
\hline Estado Excelente & $>6$ & 7 & $<5$ \\
\hline Estado Bom & $>6$ a 5 & Entre 6,5 e 8,5 & 5 \\
\hline Estado Razoável & $>5$ a 4 & Entre 5,5 e 6,5 / Entre 8,5 e 9,5 & $>5$ a 20 \\
\hline Estado Insatisfatório & $>4$ a 3 & Entre 4,5 e 5,5 / Entre 9,5 e 10,5 & $>20$ a 40 \\
\hline Estado Ruim & $<3$ & $<4,5 />10,5$ & $>40$ \\
\hline
\end{tabular}

Tabela 4. Variáveis analisadas: compostos nitrogenados.

\begin{tabular}{|c|c|c|c|}
\hline \multirow{2}{*}{$\begin{array}{l}\text { Qualidade } \\
\text { Ecológica }\end{array}$} & \multicolumn{3}{|c|}{ Ponderação } \\
\hline & Amônia & Nitrito & Nitrato \\
\hline Estado Excelente & $<0,40$ mg.L-1 & $<0,07 \mathrm{mg} \cdot \mathrm{L}^{-1}$ & $<0,40$ mg.L-1 \\
\hline Estado Bom & 0,40 mg.L-1 & 0,07 mg.L ${ }^{-1}$ & 0,40 mg.L-1 \\
\hline Estado Razoável & $>0,40$ a 1 mg.L-1 & $>0,07$ a $0,1 \mathrm{mg} . \mathrm{L}-1$ & $>0,40$ a $1 \mathrm{mg} \cdot \mathrm{L}-1$ \\
\hline Estado Insatisfatório & $>1$ a 2 mg.L-1 & $>1$ a 1,3 mg.L-1 & $>1$ a 2 mg.L-1 \\
\hline Estado Ruim & $>3$ mg.L-1 & $>1,3$ mg.L-1 & $>3 \mathrm{mg} \cdot \mathrm{L}-1$ \\
\hline
\end{tabular}

As amostras foram quantificadas, divididas e dispostas em tabelas que indicam as ponderações de acordo com o supracitado (Tabelas 3 e 4). Para a obtenção e análise dos resultados foram apresentadas novas tabelas que correlacionam o número de amostras com as ponderações referentes à Qualidade Ecológica. Além disso, foi calculada a porcentagem de amostras dispostas em cada intervalo. Cabe destacar que para o cálculo das porcentagens foi utilizada a função arredondar para duas casas decimais do software Microsoft Excel [=ARRED (célula; 2)]. A função ARRED arredonda um número para um número especificado de dígitos. Por exemplo, se a célula contiver 0,1725 esse valor será arredondado para 0,17 e será apresentado como $17 \%$.

\section{Resultados}

\section{Oxigênio Dissolvido (OD)}

As concentrações de OD na superfície ficaram distribuídas entre um mínimo de 0,09 mg.L-1 e um máximo de 8,84 mg.L. . $^{-1}$ Próximo ao fundo as concentrações de OD variaram de de 0,08 a 10,39 mg. $\mathrm{L}^{-1}$. 
Quando submetido à DQA, a maior parte das análises (60\%) para o parâmetro de OD para a superfície está no estado ruim (Tabela 5), indicando alterações graves dos valores dos elementos de qualidade ecológica. Neste estado os elementos mais afetados são o fitoplâncton, macrófitas e fitobentos, invertebrados bentônicos e fauna piscícola, que podem apresentar modificações no na composição e abundância.

No que se refere à análise de fundo para OD, 55\% das amostras foram classificadas com ruim, quando aplicada a DQA (Tabela 5), indicando que durante o período de monitoramento a qualidade ecológica da água apresentou alterações significativas em relação aos limites estabelecidos pela Resolução CONAMA no 375/2005.

Tabela 5. Percentual de amostras para OD.

\begin{tabular}{|c|c|c|c|c|}
\hline Qualidade Ecológica & $\begin{array}{c}\text { No de amostras na } \\
\text { Superfície }\end{array}$ & $\mathbf{\%}$ & $\begin{array}{c}\text { No de amostras } \\
\text { no fundo }\end{array}$ & $\mathbf{\%}$ \\
\hline Estado Excelente & 59 & $17 \%$ & 45 & $13 \%$ \\
\hline Estado Bom & 1 & $0 \%$ & 0 & $0 \%$ \\
\hline Estado Razoável & 46 & $14 \%$ & 38 & $11 \%$ \\
\hline Estado Insatisfatório & 32 & $9 \%$ & 71 & $21 \%$ \\
\hline Estado Ruim & 202 & $60 \%$ & 186 & $55 \%$ \\
\hline TOTAL & $\mathbf{3 4 0}$ & $\mathbf{1 0 0 \%}$ & $\mathbf{3 4 0}$ & $\mathbf{1 0 0 \%}$ \\
\hline
\end{tabular}

$\mathrm{Na}$ análise por ponto de monitoramento de superfície, é possível observar, que as estações PM3 e PM4 obtiveram os melhores resultados com 17 e 33 amostras, respectivamente, acima do preconizado na Resolução CONAMA n $375 / 2005$, sendo classificados como excelente (Tabela 6).

Tabela 6. Classificação das amostras para OD por ponto de monitoramento.

\begin{tabular}{|c|c|c|c|c|c|c|c|c|c|c|c|}
\hline \multirow{3}{*}{ Qualidade Ecológica } & \multirow{3}{*}{ Ponderação } & \multicolumn{5}{|c|}{ Superfície } & \multicolumn{5}{|c|}{ Fundo } \\
\hline & & \multirow{2}{*}{$\begin{array}{c}\text { № de } \\
\text { amostras }\end{array}$} & \multicolumn{4}{|c|}{ Ponto } & \multirow{2}{*}{$\begin{array}{c}\text { № de } \\
\text { amostras }\end{array}$} & \multicolumn{4}{|c|}{ Ponto } \\
\hline & & & PM1 & PM2 & PM3 & PM4 & & PM1 & PM2 & PM3 & PM4 \\
\hline Estado Excelente & $>5 \mathrm{mg} \cdot \mathrm{L}^{-1}$ & 59 & 4 & 5 & 17 & 33 & 45 & 7 & 7 & 18 & 13 \\
\hline Estado Bom & $5 \mathrm{mg} \cdot \mathrm{L}^{-1}$ & 1 & 0 & 1 & 0 & 0 & 0 & 0 & 0 & 0 & 0 \\
\hline Estado Razoável & $>5$ a $4 \mathrm{mg} \cdot \mathrm{L}^{-1}$ & 46 & 4 & 9 & 11 & 22 & 38 & 6 & 8 & 8 & 16 \\
\hline Estado Insatisfatório & $>4$ a 3 mg. $\mathrm{L}^{-1}$ & 32 & 6 & 2 & 8 & 16 & 71 & 15 & 19 & 13 & 24 \\
\hline Estado Ruim & $<3 \mathrm{mg} \cdot \mathrm{L}^{-1}$ & 202 & 71 & 68 & 49 & 14 & 186 & 57 & 51 & 46 & 32 \\
\hline Tota & & 340 & 85 & 85 & 85 & 85 & 340 & 85 & 85 & 85 & 85 \\
\hline
\end{tabular}


No que se refere à análise ponto a ponto para o monitoramento de fundo, as estações que apresentaram melhor qualidade ambiental foram as PM3 e PM4, com 18 e 13 amostras, respectivamente, classificadas como excelente de acordo com a DQA. Ressaltase, ainda, que também foram verificadas amostras classificadas como excelente nas demais estações.

0 resultado das análises das amostras de superfície e de fundo demonstra que a qualidade da água em relação ao OD está abaixo do limite preconizado pela Resolução CONAMA no $375 / 2005$, sendo mais da metade delas (60\% de superfície e $55 \%$ de fundo) classificadas como ruim pela DQA. Os pontos com melhor qualidade são os pontos PM3 e PM4, localizados na área mais externa do Canal do Cunha, pois tem maior renovação de água costeira devido à influência da maré.

\section{pH}

Os resultados de $\mathrm{pH}$ na camada superficial variaram de 6,96 a 9,61. $\mathrm{Na}$ camada próxima ao fundo os valores de pH ficaram entre 7,09 e 9,55.

$0 \mathrm{pH}$ está relacionado com $\mathrm{o}$ equilíbrio ácido base dos processos biológicos e pode ser um indicativo de mudanças no estado fisiológico de um determinado ambiente (poluição e eutrofização) (Paranhos, 1996). 0 pH da água do mar está geralmente entre 7,5 e 8,4 e as modificações nesse parâmetro raramente representam um fator limitante. A respiração, fotossíntese, trocas ar-oceano e precipitação são alguns dos fatores que podem provocar mudanças fisiológicas, causando alterações do pH (Paranhos, 1996).

De acordo com os dados do INEA, o valor de pH medido na estação CN100, localizada próxima ao local do monitoramento, no dia 10 de novembro de 2015, foi de 6,8 (INEA, 2015). Este valor é inferior ao menor valor $(6,96)$ encontrado para este parâmetro ao longo deste monitoramento.

Quanto submetidos ao DQA, 92\% dos parâmetros obtidos para a superfície apresentaram classificação "estado bom", de acordo com a metodologia aplicada (Tabela 7). Vale lembrar, que a qualidade ambiental excelente foi considerada para o pH neutro, sendo exatamente 7 .

Os índices observados para o fundo são ainda melhores, que para a superfície, apresentando $96 \%$ das amostras na classificação "Estado Bom" quando aplicada a DQA (Tabela 7), que pode está relacionado ao fato de os valores de $\mathrm{pH}$ variarem de acordo com os fatores naturais, tais como a absorção de gases atmosféricos, oxidação da matéria orgânica e fotossíntese (Von Sperling, 2005).

Tabela 7. Percentual de amostras para $\mathrm{pH}$.

\begin{tabular}{|c|c|c|c|c|}
\hline \multirow{2}{*}{ Qualidade Ecológica } & \multicolumn{2}{|c|}{ Superfície } & \multicolumn{2}{c|}{ Fundo } \\
\cline { 2 - 5 } & No de amostras & \% & No de amostras & $\%$ \\
\hline Estado Excelente & 0 & $0 \%$ & 0 & $0 \%$ \\
\hline Estado Bom & 313 & $92 \%$ & 326 & $96 \%$ \\
\hline Estado Razoável & 27 & $8 \%$ & 14 & $4 \%$ \\
\hline Estado Insatisfatório & 0 & $0 \%$ & 0 & $0 \%$ \\
\hline Estado Ruim & 0 & $0 \%$ & 0 & $0 \%$ \\
\hline TOTAL & $\mathbf{3 4 0}$ & $\mathbf{1 0 0 \%}$ & $\mathbf{3 4 0}$ & $\mathbf{1 0 0 \%}$ \\
\hline
\end{tabular}


Os valores de $\mathrm{pH}$, de maneira geral, apresentaram índices encontrados em ambientes similares. Essas concentrações garantem o funcionamento do ecossistema da Baía de Guanabara quando discutido o parâmetro $\mathrm{pH}$. Os piores índices para superfície, classificado como "estado razoável", foram encontrados na estação PM4, nas outras estações os índices foram similares, mas é possível observar uma qualidade melhor na PM3 (Tabela 8). Quando se observa os resultados das análises de $\mathrm{pH}$ ponto a ponto para as amostras de fundo, verifica-se que não há variações relevantes entre os pontos de monitoramento.

Dessa forma, é possível afirmar, que a atividade de dragagem não afetou de maneira significativa a qualidade da água em relação ao $\mathrm{pH}$. Apenas $8 \%$ das amostras de superfície e $4 \%$ das amostras de fundo tiveram resultado fora dos parâmetros determinado pela Resolução CONAMA no 375/2005. Quando aplicada a metodologia da DQA não foram observadas amostras consideradas como "Estado Insatisfatório" ou "Estado Ruim".

Tabela 9. Classificação das amostras para pH por ponto de monitoramento.

\begin{tabular}{|c|c|c|c|c|c|c|c|c|c|c|c|}
\hline \multirow{3}{*}{$\begin{array}{l}\text { Qualidade } \\
\text { Ecológica }\end{array}$} & \multirow{3}{*}{ Ponderação } & \multicolumn{5}{|c|}{ Superfície } & \multicolumn{5}{|c|}{ Fundo } \\
\hline & & \multirow{2}{*}{$\begin{array}{c}\text { № de } \\
\text { amostras }\end{array}$} & \multicolumn{4}{|c|}{ Ponto } & \multirow{2}{*}{$\begin{array}{c}\text { № de } \\
\text { amostras }\end{array}$} & \multicolumn{4}{|c|}{ Ponto } \\
\hline & & & PM1 & PM2 & PM3 & PM4 & & PM1 & PM2 & PM3 & PM4 \\
\hline Estado Excelente & 7 & 0 & 0 & 0 & 0 & 0 & 0 & 0 & 0 & 0 & 0 \\
\hline Estado Bom & Entre 6,5 e 8,5 & 313 & 80 & 81 & 82 & 70 & 326 & 82 & 81 & 82 & 81 \\
\hline Estado Razoável & $\begin{array}{c}\text { Entre } 5,5 \text { e } 6,5 / \\
\text { Entre } 8,5 \text { e } 9,5\end{array}$ & 27 & 5 & 4 & 3 & 15 & 14 & 3 & 4 & 3 & 4 \\
\hline Estado Insatisfatório & $\begin{array}{l}\text { Entre } 4,5 \text { e } 5,5 / \\
\text { Entre } 9,5 \text { e } 10,5\end{array}$ & 0 & 0 & 0 & 0 & 0 & 0 & 0 & 0 & 0 & 0 \\
\hline Estado Ruim & $<4,5 />10,5$ & 0 & 0 & 0 & 0 & 0 & 0 & 0 & 0 & 0 & 0 \\
\hline Total & & 340 & 85 & 85 & 85 & 85 & 340 & 85 & 85 & 85 & 85 \\
\hline
\end{tabular}

\section{amônia}

\section{Compostos nitrogenados -}

Os resultados das amostras coletadas indicam que níveis de amônia foram mais altos na camada de superfície, sendo o valor mínimo de 0,37 e o máximo, 9,08 mg. $\mathrm{L}^{-1}$.

Quando os resultados do monitoramento foram submetidos ao DQA, foi possível observar, que $52 \%$ das amostras de superfície para amônia estão em estado ruim, de acordo com a metodologia adotada (Tabela 9). Percentualmente a qualidade das amostras de fundo é melhor que a qualidade das águas da superfície, mas ainda assim acima dos limites estabelecidos pela Resolução CONAMA no $375 / 2005$, desta forma, sendo classificados como estado razoável (59\%), estado insatisfatório (26\%) e estado ruim (5\%), conforme observado na Tabela 9. 
Tabela 9. Percentual de amostras para amônia.

\begin{tabular}{|c|c|c|c|c|}
\hline \multirow{2}{*}{ Qualidade Ecológica } & \multicolumn{2}{|c|}{ Superfície } & \multicolumn{2}{c|}{ Fundo } \\
\cline { 2 - 5 } & No de amostras & $\mathbf{\%}$ & No de amostras & \% \\
\hline Estado Excelente & 3 & $3 \%$ & 9 & $10 \%$ \\
\hline Estado Bom & 0 & $0 \%$ & 0 & $0 \%$ \\
\hline Estado Razoável & 9 & $10 \%$ & 52 & $59 \%$ \\
\hline Estado Insatisfatório & 31 & $35 \%$ & 23 & $26 \%$ \\
\hline Estado Ruim & 45 & $52 \%$ & 4 & $5 \%$ \\
\hline TOTAL & $\mathbf{8 8}$ & $\mathbf{1 0 0 \%}$ & $\mathbf{8 8}$ & $\mathbf{1 0 0 \%}$ \\
\hline
\end{tabular}

$\mathrm{Na}$ análise por ponto de monitoramento para superfície, a estação PM4 apresentou os melhores resultados, tendo, inclusive, três amostras classificadas como excelentes. Já a estação PM1 apresentou os piores resultados, sendo 19 amostras classificadas como estado ruim (Tabela 10). Já as amostras de fundo indicaram que o ponto com melhor qualidade ambiental, de acordo com metodologia aplicada, é o ponto PM4, apresentando três amostras com estado excelente.

As amostras de superfície têm piores classificações de DQA quando comparados às amostras de fundo, apresentando alterações significativas em relação a estes parâmetros, no entanto, nas duas profundidades os piores pontos são os das estações PM1 e PM2.

Tabela 10. Classificação das amostras para amônia por ponto de monitoramento.

\begin{tabular}{|c|c|c|c|c|c|c|c|c|c|c|c|}
\hline \multirow{3}{*}{ Qualidade Ecológica } & \multirow{3}{*}{ Ponderação } & \multicolumn{5}{|c|}{ Superfície } & \multicolumn{5}{|c|}{ Fundo } \\
\hline & & \multirow{2}{*}{$\begin{array}{c}\text { № de } \\
\text { amostras }\end{array}$} & \multicolumn{4}{|c|}{ Ponto } & \multirow{2}{*}{$\begin{array}{c}\text { № de } \\
\text { amostras }\end{array}$} & \multicolumn{4}{|c|}{ Ponto } \\
\hline & & & PM1 & PM2 & PM3 & PM4 & & PM1 & PM2 & PM3 & PM4 \\
\hline Estado Excelente & $<0,40 \mathrm{mg} \cdot \mathrm{L}^{-1}$ & 3 & 0 & 0 & 0 & 3 & 3 & 0 & 0 & 0 & 3 \\
\hline Estado Bom & $0,40 \mathrm{mg} \cdot \mathrm{L}^{-1}$ & 0 & 0 & 0 & 0 & 0 & 0 & 0 & 0 & 0 & 0 \\
\hline Estado Razoável & $>0,40$ a $1 \mathrm{mg} \cdot \mathrm{L}^{-1}$ & 9 & 0 & 0 & 2 & 7 & 9 & 0 & 0 & 2 & 7 \\
\hline Estado Insatisfatório & $>1$ a 2 mg. $\mathrm{L}^{-1}$ & 31 & 3 & 10 & 8 & 10 & 31 & 3 & 10 & 8 & 10 \\
\hline Estado Ruim & $>3$ mg.L-1 & 45 & 19 & 12 & 12 & 2 & 45 & 19 & 12 & 12 & 2 \\
\hline \multicolumn{2}{|c|}{ Total } & 88 & 22 & 22 & 22 & 22 & 88 & 22 & 22 & 22 & 22 \\
\hline
\end{tabular}

\section{nitrito}

\section{Compostos nitrogenados -}

A variação dos valores de nitrito ao longo do monitoramento foi de abaixo do limite de detecção $\left(<0,00014 \mathrm{mg} \cdot \mathrm{L}^{-1}\right) \mathrm{a}$ 0,074 mg. $\mathrm{L}^{-1}$.

Ao classificar a qualidade ecológica da água de acordo com a DQA, 
tendo como referência os valores máximos para nitrito estabelecido pela Resolução CONAMA no 375/2005, 87 das 88 (ou seja, 99\%) amostras de superfície foram classificadas como "Estado
Excelente". No monitoramento de fundo, $100 \%$ das amostras foram classificadas como "Estado Excelente" em todos os pontos de monitoramento (Tabela 11).

Tabela 11. Percentual de amostras para nitrito.

\begin{tabular}{|c|c|c|c|c|}
\hline \multirow{2}{*}{ Qualidade Ecológica } & \multicolumn{2}{|c|}{ Superfície } & \multicolumn{2}{c|}{ Fundo } \\
\cline { 2 - 5 } & № de amostras & $\mathbf{\%}$ & № de amostras & \% \\
\hline Estado Excelente & 87 & $99 \%$ & 88 & $100 \%$ \\
\hline Estado Bom & 0 & $0 \%$ & 0 & $0 \%$ \\
\hline Estado Razoável & 1 & $1 \%$ & 0 & $0 \%$ \\
\hline Estado Insatisfatório & 0 & $0 \%$ & 0 & $0 \%$ \\
\hline Estado Ruim & 0 & $0 \%$ & 0 & $0 \%$ \\
\hline TOTAL & $\mathbf{8 8}$ & $\mathbf{1 0 0 \%}$ & $\mathbf{8 8}$ & $\mathbf{1 0 0 \%}$ \\
\hline
\end{tabular}

$\mathrm{Na}$ análise por ponto de monitoramento e, conforme já citado, apenas uma amostra de superfície diferiu das demais e foi classificada como "Estado Razoável" para o ponto PM4 (Tabela 12).

0 nitrito não variou significativamente entre a camada superficial e a próxima do fundo, tampouco entre as estações de monitoramento, permanecendo quase em sua totalidade como "Estado Excelente" durante todo o período de monitoramento da área de dragagem.

Tabela 12. Classificação das amostras para nitrito por ponto de monitoramento

\begin{tabular}{|c|c|c|c|c|c|c|c|c|c|c|c|}
\hline \multirow{3}{*}{$\begin{array}{l}\text { Qualidade } \\
\text { Ecológica }\end{array}$} & \multirow{3}{*}{ Ponderação } & \multicolumn{5}{|c|}{ Superfície } & \multicolumn{5}{|c|}{ Fundo } \\
\hline & & \multirow{2}{*}{$\begin{array}{c}\text { № de } \\
\text { amostras }\end{array}$} & \multicolumn{4}{|c|}{ Ponto } & \multirow{2}{*}{$\begin{array}{c}\text { № de } \\
\text { amostras }\end{array}$} & \multicolumn{4}{|c|}{ Ponto } \\
\hline & & & PM1 & PM2 & PM3 & PM4 & & PM1 & PM2 & PM3 & PM4 \\
\hline Estado Excelente & $<0,07 \mathrm{mg} \cdot \mathrm{L}^{-1}$ & 87 & 22 & 22 & 22 & 21 & 88 & 22 & 22 & 22 & 22 \\
\hline Estado Bom & $0,07 \mathrm{mg} \cdot \mathrm{L}^{-1}$ & 0 & 0 & 0 & 0 & 0 & 0 & 0 & 0 & 0 & 0 \\
\hline Estado Razoável & $>0,07$ a $0,1 \mathrm{mg} \cdot \mathrm{L}-1$ & 1 & 0 & 0 & 0 & 1 & 0 & 0 & 0 & 0 & 0 \\
\hline Estado Insatisfatório & $>1$ a 1,3 mg.L-1 & 0 & 0 & 0 & 0 & 0 & 0 & 0 & 0 & 0 & 0 \\
\hline Estado Ruim & $>1,3$ mg.L-1 & 0 & 0 & 0 & 0 & 0 & 0 & 0 & 0 & 0 & 0 \\
\hline \multicolumn{2}{|c|}{ Total } & 88 & 22 & 22 & 22 & 22 & 88 & 22 & 22 & 22 & 22 \\
\hline
\end{tabular}



nitrato

\section{Compostos nitrogenados -}

Os resultados das amostras para os níveis de nitrato próximo ao fundo foram mais altos que na superfície. As concentrações na superfície variaram desde abaixo do limite de detecção $\left(<0,0007 \mathrm{mg} . \mathrm{L}^{-1}\right)$ a $0,068 \mathrm{mg} . \mathrm{L}^{-1}$. Próximo ao fundo, as concentrações também variaram desde abaixo do limite de detecção $\left(<0,0007\right.$ mg. $\left.L^{-1}\right)$ a 0,537 mg.L-1 .
Quando os aplicados os índices de DQA a partir dos resultados dos monitoramentos, as classificações de nitrato não tiveram diferença, apresentando a qualidade ambiental classificado como "Estado Excelente" (Tabela 13). Cabe destacar, que esse resultado era esperado, uma vez que todas as amostras se mantiveram abaixo do limite estabelecidos pela Resolução CONAMA no $375 / 2005$ para o parâmetro nitrato.

Tabela 13. Percentual de amostras para nitrato.

\begin{tabular}{|c|c|c|c|c|}
\hline \multirow{2}{*}{ Qualidade Ecológica } & \multicolumn{2}{|c|}{ Superfície } & \multicolumn{2}{c|}{ Fundo } \\
\cline { 2 - 5 } & No de amostras & $\mathbf{\%}$ & No de amostras & \% \\
\hline Estado Excelente & 88 & $100 \%$ & 88 & $100 \%$ \\
\hline Estado Bom & 0 & $0 \%$ & 0 & $0 \%$ \\
\hline Estado Razoável & 0 & $0 \%$ & 0 & $0 \%$ \\
\hline Estado Insatisfatório & 0 & $0 \%$ & 0 & $0 \%$ \\
\hline Estado Ruim & 0 & $0 \%$ & 0 & $0 \%$ \\
\hline TOTAL & $\mathbf{8 8}$ & $\mathbf{1 0 0 \%}$ & $\mathbf{8 8}$ & $\mathbf{1 0 0 \%}$ \\
\hline
\end{tabular}

Tabela 14. Classificação das amostras para nitrato por ponto de monitoramento.

\begin{tabular}{|c|c|c|c|c|c|c|c|c|c|c|c|}
\hline \multirow{3}{*}{$\begin{array}{l}\text { Qualidade } \\
\text { Ecológica }\end{array}$} & \multirow{3}{*}{ Ponderação } & \multicolumn{5}{|c|}{ Superfície } & \multicolumn{5}{|c|}{ Fundo } \\
\hline & & \multirow{2}{*}{$\begin{array}{c}\text { № de } \\
\text { amostras }\end{array}$} & \multicolumn{4}{|c|}{ Ponto } & \multirow{2}{*}{$\begin{array}{c}\text { № de } \\
\text { amostras }\end{array}$} & \multicolumn{4}{|c|}{ Ponto } \\
\hline & & & PM1 & PM2 & PM3 & PM4 & & PM1 & PM2 & PM3 & PM4 \\
\hline Estado Excelente & $<0,40 \mathrm{mg} \cdot \mathrm{L}^{-1}$ & 88 & 22 & 22 & 22 & 22 & 88 & 22 & 22 & 22 & 22 \\
\hline Estado Bom & 0,40 mg. $L^{-1}$ & 0 & 0 & 0 & 0 & 0 & 0 & 0 & 0 & 0 & 0 \\
\hline Estado Razoável & $>0,40$ a $1 \mathrm{mg} \cdot \mathrm{L}^{-1}$ & 0 & 0 & 0 & 0 & 0 & 0 & 0 & 0 & 0 & 0 \\
\hline $\begin{array}{c}\text { Estado } \\
\text { Insatisfatório }\end{array}$ & $>1$ a 2 mg. $L^{-1}$ & 0 & 0 & 0 & 0 & 0 & 0 & 0 & 0 & 0 & 0 \\
\hline Estado Ruim & $>3 \mathrm{mg} \cdot \mathrm{L}^{-1}$ & 0 & 0 & 0 & 0 & 0 & 0 & 0 & 0 & 0 & 0 \\
\hline \multicolumn{2}{|c|}{ Total } & 88 & 22 & 22 & 22 & 22 & 88 & 22 & 22 & 22 & 22 \\
\hline
\end{tabular}

\section{Turbidez}

Os valores de turbidez variaram de 0,05 a 143,33 UNT na superfície. Próximo ao fundo os valores de turbidez variaram entre 0,89 e 103,22 UNT.

A turbidez variou significativamente entre a superfície e o fundo e ao longo dos meses monitorados. De acordo com o INEA, o valor de turbidez medido no canal do Cunha (estação CN100), em novembro de 2015, foi de 2,70 UNT (INEA, 2015). Conforme discutido anteriormente, a Resolução CONAMA no $357 / 2005$ não estabelece nenhum padrão para o parâmetro turbidez.

Quando submetido à DQA, a maior parte das análises para superfície (45\%) para o parâmetro de turbidez está no estado insatisfatório (Tabela 15), indicando presença de materiais em 
suspensão. No que se refere à análise de fundo para turbidez, $66 \%$ das amostras foram classificadas como razoável, quando aplicada a DQA (Tabela 15), ainda indicando a necessidade de ações, mas com parâmetros melhores quando comparados com a turbidez de superfície.

Tabela 15. Percentual de amostras para turbidez.

\begin{tabular}{|c|c|c|c|c|}
\hline \multirow{2}{*}{ Qualidade Ecológica } & \multicolumn{2}{|c|}{ Superfície } & \multicolumn{2}{c|}{ Fundo } \\
\cline { 2 - 5 } & $\begin{array}{c}\text { No de } \\
\text { amostras }\end{array}$ & $\mathbf{\%}$ & $\begin{array}{c}\text { No de } \\
\text { amostras }\end{array}$ & $\mathbf{\%}$ \\
\hline Estado Excelente & 7 & $2 \%$ & 88 & $26 \%$ \\
\hline Estado Bom & 0 & $0 \%$ & 0 & $0 \%$ \\
\hline Estado Razoável & 88 & $26 \%$ & 223 & $66 \%$ \\
\hline Estado Insatisfatório & 152 & $45 \%$ & 24 & $7 \%$ \\
\hline Estado Ruim & 93 & $27 \%$ & 5 & $1 \%$ \\
\hline TOTAL & $\mathbf{3 4 0}$ & $\mathbf{1 0 0 \%}$ & $\mathbf{3 4 0}$ & $\mathbf{1 0 0 \%}$ \\
\hline
\end{tabular}

$\mathrm{Na}$ análise por ponto de monitoramento na superfície, é possível observar, que as estações PM1 e PM2 obtiveram os piores resultados com $45 \mathrm{e}$ 30 amostras, respectivamente, classificadas como no Estado Ruim (Tabela 16). Para o fundo, analisando ponto a ponto, as estações que apresentaram pior qualidade ambiental foram novamente as estações PM1 e PM2, com 13 e 10 amostras, respectivamente, classificadas como insatisfatório e duas e três amostras, respectivamente, classificadas como ruim de acordo com a DQA.

0 resultado das análises das amostras de superfície e de fundo demonstra que houve uma ressuspensão de sedimentos e indicam necessidade de ações de acordo com a DQA. De maneira geral, os maiores valores de turbidez ocorreram nas estações PM1 e PM2. As estações PM3 e PM4, mais próximas da entrada de água pelo canal Central, apresentaram valores de turbidez menores que as outras duas estações.

Tabela 16. Classificação das amostras para turbidez por ponto de monitoramento .

\begin{tabular}{|c|c|c|c|c|c|c|c|c|c|c|c|}
\hline \multirow{3}{*}{$\begin{array}{l}\text { Qualidade } \\
\text { Ecológica }\end{array}$} & \multirow{3}{*}{ Ponderação } & \multicolumn{5}{|c|}{ Superfície } & \multicolumn{5}{|c|}{ Fundo } \\
\hline & & \multirow{2}{*}{$\begin{array}{c}\text { № de } \\
\text { amostras }\end{array}$} & \multicolumn{4}{|c|}{ Ponto } & \multirow{2}{*}{$\begin{array}{c}\text { № de } \\
\text { amostras }\end{array}$} & \multicolumn{4}{|c|}{ Ponto } \\
\hline & & & PM1 & PM2 & PM3 & PM4 & & PM1 & PM2 & PM3 & PM4 \\
\hline Estado Excelente & $<5$ UNT & 7 & 1 & 1 & 0 & 5 & 88 & 12 & 4 & 30 & 42 \\
\hline Estado Bom & 5 UNT & 0 & 0 & 0 & 0 & 0 & 0 & 0 & 0 & 0 & 0 \\
\hline Estado Razoável & $>5$ a 20 UTN & 88 & 11 & 15 & 18 & 44 & 223 & 58 & 68 & 54 & 43 \\
\hline $\begin{array}{c}\text { Estado } \\
\text { Insatisfatório }\end{array}$ & $>20$ a 40 UNT & 152 & 28 & 39 & 52 & 33 & 24 & 13 & 10 & 1 & 0 \\
\hline Estado Ruim & $>40$ UNT & 93 & 45 & 30 & 15 & 3 & 5 & 2 & 3 & 0 & 0 \\
\hline To & & 340 & 85 & 85 & 85 & 85 & 340 & 85 & 85 & 85 & 85 \\
\hline
\end{tabular}


amostras

Resumo da classificação das

Foi realizado um total de 2.568 análises dos seguintes parâmetros: Oxigênio dissolvido, pH, Amônia, Nitrito, Nitrato e Turbidez. Conforme abordado, apesar de não haver um limite indicado na Resolução CONAMA, a turbidez foi incluída nas análises por ser um indicador de possíveis alterações relacionadas às atividades de dragagem. Desse total, 1.202 das análises foram classificadas como estando no Estado Excelente ou Bom, ou seja, estão no estado desejado. Já 1.366 das análises foram classificadas como estando nos Estados Razoável, Insatisfatório ou Ruim, o que de acordo com a DQA indica a necessidade de ações no corpo hídrico (Tabela 17).

Tabela 17. Número total de amostras.

\begin{tabular}{|c|c|}
\hline Qualidade Ecológica & № de Amostras \\
\hline Estado Excelente & 562 \\
\hline Estado Bom & 640 \\
\hline Estado Razoável & 498 \\
\hline Estado Insatisfatório & 333 \\
\hline Estado Ruim & 535 \\
\hline TOTAL & $\mathbf{2 . 5 6 8}$ \\
\hline
\end{tabular}

Tabela 18. Número Total de Amostras em porcentagens.

\begin{tabular}{|c|l|c|c|c|c|c|}
\hline \multicolumn{2}{|c|}{ Parâmetros } & \multicolumn{5}{c|}{ Qualidade Ecológica } \\
\cline { 2 - 7 } Oxigênio Dissolvido & Excelente & Bom & Razoável & Insatisfatório & Ruim \\
\cline { 2 - 7 } & Fuperfície & $17 \%$ & $0 \%$ & $14 \%$ & $9 \%$ & $60 \%$ \\
\hline \multirow{2}{*}{$\mathbf{p H}$} & Fundo & $13 \%$ & $0 \%$ & $11 \%$ & $21 \%$ & $55 \%$ \\
\cline { 2 - 7 } & Fuperfície & $0 \%$ & $92 \%$ & $8 \%$ & $0 \%$ & $0 \%$ \\
\hline \multirow{2}{*}{ Turbidez } & Superfície & $2 \%$ & $0 \%$ & $26 \%$ & $4 \%$ & $0 \%$ \\
\cline { 2 - 7 } & Fundo & $26 \%$ & $0 \%$ & $66 \%$ & $7 \%$ & $27 \%$ \\
\hline \multirow{2}{*}{ Amônia } & Superfície & $3 \%$ & $0 \%$ & $10 \%$ & $35 \%$ & $52 \%$ \\
\cline { 2 - 7 } & Fundo & $10 \%$ & $0 \%$ & $59 \%$ & $26 \%$ & $5 \%$ \\
\hline \multirow{2}{*}{ Nitrito } & Superfície & $99 \%$ & $0 \%$ & $1 \%$ & $0 \%$ & $0 \%$ \\
\cline { 2 - 7 } & Fundo & $100 \%$ & $0 \%$ & $0 \%$ & $0 \%$ & $0 \%$ \\
\hline \multirow{2}{*}{ Nitrato } & Superfície & $100 \%$ & $0 \%$ & $0 \%$ & $0 \%$ & $0 \%$ \\
\cline { 2 - 7 } & Fundo & $100 \%$ & $0 \%$ & $0 \%$ & $0 \%$ & $0 \%$ \\
\hline
\end{tabular}

Destacam-se as análises de nitrito (fundo) e nitrato (superfície e fundo), que obtiveram $100 \%$ das amostras classificadas como excelente, além da análise de nitrito (superfície) com 99\% das amostras também no estado excelente.

Dentre os resultados negativos, é possível destacar o oxigênio dissolvido com $60 \%$ das amostras de superfície e
$55 \%$ das amostras de fundo classificadas como no estado ruim.

A DQA realiza uma abordagem ecológica caracterizando as variáveis físicas e químicas da água, entretanto esta classificação é apenas uma das ferramentas da DQA. A Tabela 19 apresenta essa abordagem e indica a necessidade da aplicação de medidas mitigadoras, de acordo com a metodologia adotada. 
Tabela 19. Classificação das medidas mitigadoras.

\begin{tabular}{|l|l|c|c|}
\hline \multirow{2}{*}{ Variáveis } & \multicolumn{2}{|c|}{ Medidas } \\
\cline { 3 - 4 } Oxigênio dissolvido & Superfície & $17 \%$ & Necessita de ações \\
\cline { 2 - 4 } & Fundo & $13 \%$ & $83 \%$ \\
\hline \multirow{2}{*}{ pH } & Superfície & $92 \%$ & $87 \%$ \\
\cline { 2 - 4 } & Fundo & $96 \%$ & $8 \%$ \\
\hline \multirow{2}{*}{ Turbidez } & Superfície & $2 \%$ & $4 \%$ \\
\cline { 2 - 4 } & Fundo & $26 \%$ & $98 \%$ \\
\hline \multirow{2}{*}{ Amônia } & Superfície & $3 \%$ & $74 \%$ \\
\cline { 2 - 4 } & Fundo & $10 \%$ & $97 \%$ \\
\hline \multirow{2}{*}{ Nitrito } & Superfície & $99 \%$ & $90 \%$ \\
\cline { 2 - 4 } & Fundo & $100 \%$ & $1 \%$ \\
\hline \multirow{2}{*}{ Nitrato } & Superfície & $100 \%$ & $0 \%$ \\
\cline { 2 - 4 } & Fundo & $100 \%$ & $0 \%$ \\
\hline
\end{tabular}

Durante as atividades de dragagem, as análises e classificações da DQA indicaram que, de maneira geral, as águas coletadas necessitam de ações de mitigação. Principalmente para as variáveis oxigênio dissolvido (83\% para as amostras de superfície e $87 \%$ para as de fundo), turbidez (98\% para as amostras de superfície e $74 \%$ para as de fundo) e amônia (93\% para as amostras de superfície e $90 \%$ para as de fundo). A
Directiva-Quadro da Água apresenta um conceito global para proteção e uso sustentável dos corpos d'água, pois indica, a partir da caracterização e sua função no ecossistema, quais necessitam de medidas para melhoria.

Neste contexto, $53 \%$ do total de parâmetros analisados indicam necessidade de ações como pode ser observado na Figura 2.

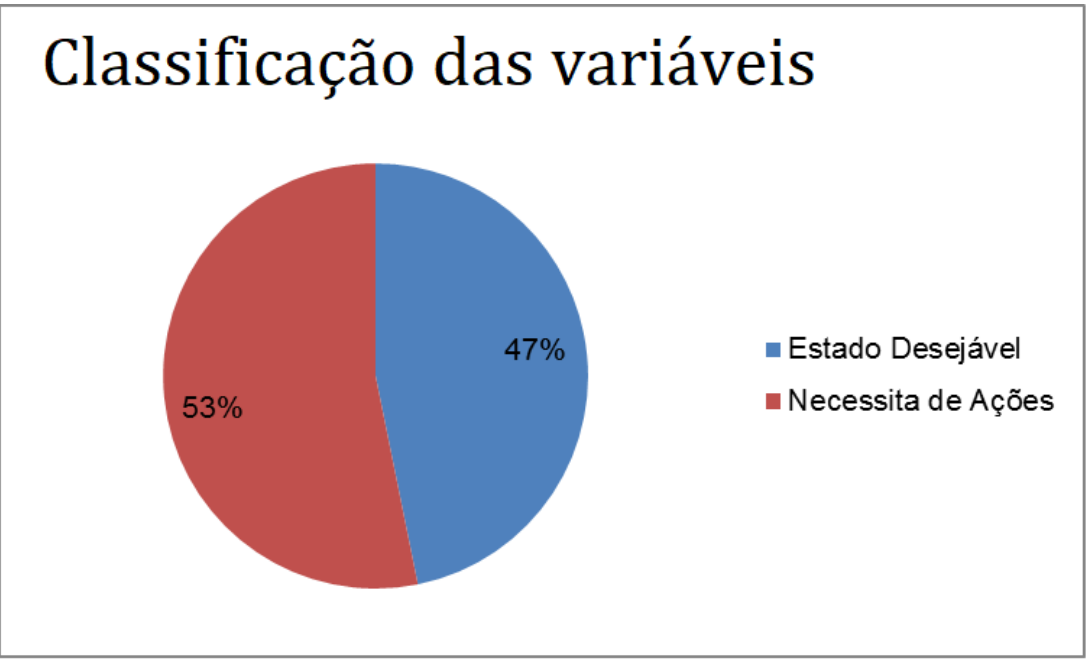

Figura 2. Classificação das variáveis estudadas. 


\section{Discussão}

O local de amostragem das estações da área de dragagem está localizado no lado noroeste da baía de Guanabara e caracteriza-se como a região mais deteriorada devido às várias fontes de poluição e aos aterros que diminuem a circulação de água na área (Mayr et al., 1989). Logo, os níveis de nutrientes são normalmente mais elevados do que no restante da Baía de Guanabara (Mayr et al., 1989; Villac, 1990; Valentin et al., 1999a e b; Paranhos e Andrade, 2012).

A concentração de OD é um indicador sensível de processos químicos e biológicos que ocorrem no mar. Os níveis de oxigênio dissolvido são utilizados como indicadores de qualidade de água, uma vez que baixas concentrações de OD estão relacionadas à poluição ou degradação ambiental (Paranhos, 1996). A maioria das concentrações de OD, tanto na superfície quanto próximo ao fundo, ficou abaixo do limite mínimo (> $5 \mathrm{mg} \cdot \mathrm{L}^{-1}$ ) estabelecido na Resolução CONAMA no 357/2005.

A poluição orgânica é uma das principais fontes de agentes contaminantes nas águas da baía de Guanabara, ocasionando um processo de degradação crescente. Este processo vem ocorrendo principalmente desde a década de 80 (Mayr et al., 1989; Villac, 1990), o que vem causando intensa eutrofização das águas da baía. A eutrofização consiste em um aumento dos sais nutrientes essenciais ao desenvolvimento dos organismos fotossintetizantes, causando uma modificação nas populações algais e aumento da proliferação das espécies. A concentração de OD mais alta na superfície pode estar relacionada com a floração de algas no período de luz em ambiente eutrofizado, ocorrendo assim uma supersaturação.

Quando submetido à DQA, a maior parte das análises (60\%) para o parâmetro de OD para a superfície está no estado ruim, indicando alterações graves dos valores dos elementos de qualidade ecológica. Neste estado os elementos mais afetados são o fitoplâncton, macrófitas e fitobentos, invertebrados bentônicos e fauna piscícola, que podem apresentar modificações no na composição e abundância.

A maioria dos valores de $\mathrm{pH}$ ficou dentro da faixa estabelecida pela Resolução CONAMA no 357/2005. O pH está relacionado ao equilíbrio ácido base dos processos biológicos e pode ser um indicativo de mudanças no estado fisiológico de um determinado ambiente (poluição e eutrofização) (Paranhos, 1996).

De acordo com os dados do INEA, o valor de pH medido na estação CN100, localizada próxima ao local do monitoramento, no dia 10 de novembro de 2015, foi de 6,8 (INEA, 2015). Este valor é inferior ao menor valor $(6,96)$ encontrado para este parâmetro ao longo deste monitoramento.

Os valores de $\mathrm{pH}$, de maneira geral, apresentaram índices encontrados em ambientes similares. Os índices observados para o fundo são melhores, quando comparado a superfície, apresentando $96 \%$ das amostras na classificação "Estado Bom" quando aplicada a DQA, que pode está relacionado ao fato de os valores de $\mathrm{pH}$ variarem de acordo com os fatores naturais, tais como a absorção de gases atmosféricos, oxidação da matéria orgânica e fotossíntese (Von Sperling, 2005).

A amônia variou significativamente entre a superfície e próximo ao fundo, enquanto o nitrato e nitrato não variaram significativamente tanto espacial quanto temporalmente. Pode-se observar que quanto maior a quantidade de amônia, mais degradado é o corpo hídrico. Já quanto há menor quantidade de amônia e maior de nitrato menos degradado é o corpo hídrico, pois ocorreram reações envolvidas no processo natural de depuração, o que significa taxas menores poluição e maior disponibilidade de OD. 
A maioria das concentrações de amônia ficou acima do limite $\left(0,40 \mathrm{mg} \cdot \mathrm{L}^{-1}\right)$ sugerido pela Resolução CONAMA no 357/2005, tanto na superfície quanto próximo ao fundo. Entretanto, estes altos valores já vem sendo observados há alguns anos na Baía de Guanabara (Paranhos e Andrade, 2012). Estes autores fizeram uma compilação de diversos estudos na Baía de Guanabara, na qual a variação da amônia encontrada foi de $<0,1$ a $80 \mathrm{mg} . \mathrm{L}^{-1}$ na área do canal central da Baía de Guanabara.

As altas concentrações encontradas para amônia podem ser explicados pelo baixo teor de oxigênio dissolvido, pela baixa velocidade do Canal do Cunha e pela grande quantidade de matéria orgânica presente na água (que consome o oxigênio dissolvido), que impede, pela ausência de oxigênio disponível, a oxidação da amônia, em primeira instância, e, posteriormente, a oxidação do nitrogênio nitrito (Amaral, 2006).

No que se refere ao nitrito, conforme informado anteriormente, os baixos valores das amostras é consequência do excesso de carga orgânica (esgoto bruto), que consome todo OD disponível, gerando elevadas taxas de demanda bioquímica de oxigênio (DBO) e de demanda química de oxigênio (DQO), não permitindo a oxidação da amônia, pela falta de oxigênio. Por outro lado, a baixa velocidade do canal não permite reaeração suficiente para dar vazão à grande carga orgânica a oxidar e a integralidade do ciclo do nitrogênio (Amaral, 2006).

Ao passo que os valores encontrados para nitrito foram baixos, espera-se o mesmo comportamento para os valores de nitrato. De acordo com Amaral (2006), isso se dá na medida em que sequer o primeiro ciclo de oxidação (amônia-nitrito) pôde ser completado pela ausência de oxigênio dissolvido, de forma que a formação do nitrato no segundo ciclo de oxidação (nitritonitrato) é ainda menor.

Analisando a turbidez, as estações localizadas próximas a entrada do canal do Cunha apresentaram os maiores valores de turbidez, região que recebe muita matéria orgânica (Figueiredo Jr. e Fernandez, 2012). Este padrão também está relacionado com a entrada de água do canal central da baía de Guanabara. Destaca-se aqui que as coletas foram realizadas durante as marés enchentes, com a entrada de água mais salina próximo ao fundo e saída de água menos salina pela superfície (Nogueira et al., 1989; Mayr et al., 1989; Kjerfve et al., 1997; Valentin, 1999a e b; Paranhos e Andrade, 2012). A turbidez foi alta devido às características ambientais já comprometidas com processos de eutrofização e degradação ambiental na área, em especial na Região do Caju próximo a influência do canal do Cunha, no entanto esse cenário é piorado durante as atividades de dragagem.

Dependendo da quantidade de partículas em suspensão na coluna de água, estas podem reduzir a transparência da água e, portanto, a produção primária. Por outro lado, elas podem servir de fonte nutritiva para os organismos zooplanctônicos (Bonecker et al., 2009).

\section{Conclusão}

$\mathrm{Na}$ área de dragagem os parâmetros medidos foram característicos de ambientes estuarinos em processo de eutrofização e degradação ambiental. No que se refere à classificação dos corpos d'água estabelecida pela legislação brasileira, a Resolução CONAMA no 357/2005 não incorporou conceitos novos de garantia da qualidade ecológica dos corpos de água, como estabelecido na União Europeia, a resolução indica os limites, mas não indica ações a serem tomadas. Assim, a aplicação da DQA poderia ser adotada em conjunto com a legislação já vigente atuando nessa indicação corpos 
hídricos que necessitam de ações ou medidas mitigadoras.

$0 \quad O D \quad$ e $\quad o \quad p H$ variaram significativamente quando considerados os parâmetros de superfície e fundo, estando os parâmetros de fundo com a pior qualidade ambiental. Entretanto, o $\mathrm{pH}$ se manteve dentro da faixa considerada "Estado Bom", enquanto o OD esteve abaixo do limite indicado na Resolução CONAMA no 357/2005, apresentado necessidades de ações.

A amônia variou significativamente entre a superfície e próximo ao fundo e apresentou valores que indicam necessidade de ações, enquanto o nitrato e nitrato não variaram significativamente tanto espacial quanto temporalmente. Destaca-se que quanto maior a quantidade de amônia, mais degradado é o corpo hídrico. Já quanto há menor quantidade de amônia e maior de nitrato menos degradado é o corpo hídrico, pois ocorreram as reações envolvidas no processo natural de depuração, o que significa taxas menores poluição e maior disponibilidade de OD. Assim, conforme já citado, a análise para amônia, indica a necessidade de ações.

Analisando a turbidez, as estações PM1 e PM2 tiveram os maiores valores de turbidez, enquanto que nas estações PM3 e PM4 foi o inverso. Este padrão também está relacionado com a entrada de água do canal central da baía de Guanabara. A turbidez pode ter sido alta devido às características ambientais já comprometidas com processos de eutrofização e degradação ambiental na área, em especial na região do Caju próximo a influência do canal do Cunha, no entanto esse cenário é piorado durante as atividades de dragagem. Portando, de acordo com a DQA, as águas analisadas necessitam de ações para esse parâmetro.

Dessa forma, de acordo com a DQA, o corpo hídrico analisado de maneira geral, necessita de ações, principalmente na região mais próxima a atividade de dragagem.

\section{Conflito de interesses}

Os autores declaram não haver conflito de interesses.

\section{Referências}

Amaral, L. C. P. Degradação ambiental e perspectivas de saúde: um olhar retrospectivo sobre a Sub-Bacia Hidrográfica do Canal do Cunha. Rio de Janeiro: Escola Nacional de Saúde Pública Sérgio Arouca, Fundação Oswaldo Cruz, 2006. (Dissertação de Mestrado em Saúde Pública).

Bonecker, A. C. T.; Bonecker, S. L. C.; Bassani, C. Plâncton marinho. In: Pereira, R. C.; SoaresGomes, A. (Orgs.). Biologia Marinha. 2. ed. Interciência: Rio de Janeiro, 2009. p. 213-239.

Brasil. Resolução CONAMA no 375 , de 17 de março de 2005. Disponível em: <http://www2.mma.gov.br/port/conama/le giabre.cfm?codlegi=459>. Acesso em: 01 ago. 2017.

Comunidades Europeia. Directiva 2000/60/CE do Parlamento Europeu e do Conselho, de 23 de outubro de 2000. Disponível em: <http://www.apambiente.pt/ dqa/assets/01-2000_60_ce---directivaquadro-da-\%c3\%a1gua.pdf>. Acesso em: Acesso em: 01 ago. 2017.

Cunha, I. A. Fronteiras da gestão: os conflitos ambientais das atividades portuárias. Revista de Administração Pública, v. 40, n. 6 , p. 1019-1040, 2006. http://doi.org/ 10.1590/S0034-76122006000600005

Figueiredo Jr., A. G.; Fernandez, G. B. Caracterização geológica e física. In: Meniconi, M. F. G.; Silva, T.; Fonseca, M. L.; Lima, S. O. F.; Lima, E. F. A.; Lavrado, H. P.; Figueiredo Júnior, A. G. (Eds.). Baía de Guanabara: síntese do conhecimento ambiental. Ambiente e influência antrópica. Rio de Janeiro: Petrobras, 2012. v. 1. p. 22-40.

INEA - Instituto Estadual do Ambiente. Boletim de qualidade das águas da região hidrográfica V - Baía de Guanabara. 2015. Disponível em <http://www.inea.rj.gov.br/ cs/groups/public/documents/document/zw ew/mte0/ edisp/inea0114695.pdf>. Acesso em: 30 abr. 2018.

Kjerfve, B.; Ribeiro, C. H. A.; Dias, G. T. M.; Filippo, A. M.; Quaresma, V.S. Oceanographic characteristics of an impacted coastal bay: Baía de Guanabara, Rio de Janeiro, Brazil. 
Continental Shelf Reserach, v. 17, n. 13, p. 1609-1643, 1997. https://doi.org/ 10.1016/S0278-4343(97)00028-9

Mayr, L. M.; Tenenbaum, D. R.; Villac, M. C.; Paranhos, R.; Nogueira, C. R.; Bonecker, S. L. C.; Bonecker, A. C. T. Hydrobiological characterization of Guanabara Bay. In: Magoon, O.; Neves, C. (Eds.). Coastlines of Brazil. New York: American Society of Civil Engineers, 1989. p. 124-138.

Nogueira, C. R.; Bonecker, A. C. T.; Bonecker, S. L. C. Zooplâncton da Baía de Guanabara (RJ - Brasil): composição e variações espaçotemporais. In: Brandini, F. P. (Ed.). Memórias do III Encontro Brasileiro de Plâncton. Caiobá, Paraná, 1989. p. 151-156.

Paranhos, R. Alguns métodos para análise da água. Cadernos Didáticos UFRJ, 1996.

Paranhos, R.; Andrade, L. Caracterização físico-química da coluna d'água e a qualidade das águas. In: Meniconi, M. F. G.; Silva, T.; Fonseca, M. L.; Lima, S. O. F.; Lima, E. F. A.; Lavrado, H. P.; Figueiredo Júnior, A. G. (Eds.). Baía de Guanabara: síntese do conhecimento ambiental. Ambiente e influência antrópica. Rio de Janeiro: Petrobras, 2012. v. 1. p. 59-79.

Valentin, J. L.; Tenenbaum, D. R.; Bonecker, A. C. T.; Bonecker, S. L. C.; Nogueira, C. R.; Villac, M. C. 0 sistema planctônico da Baía de Guanabara: síntese do conhecimento. In: Silva, S. H. G.; Lavrado, H.P. (Eds.). Ecologia dos ambientes costeiros do Estado do Rio de Janeiro. Rio de Janeiro: PPGE-UFRJ, 1999a. (Série Oecologia Brasiliensis, VII,). p. 35-59.
Valentin, J. L.; Tenenbaum, D. R.; Bonecker, A. C. T.; Bonecker, S. L. C.; Nogueira, C. R.; Paranhos, R.; Villac, M.C. Caractéristiques hydrobiologiques de la Baie de Guanabara (Rio de Janeiro, Brésil). Journal de Récherche Oceanographique, v. 24, n. 1, p. 33-41, 1999b.

Villac, M. C. O fitoplâncton como um instrumento de diagnose e monitoramento ambiental: um estudo de caso da Baía de Guanabara. Rio de Janeiro: Universidade Federal do Rio de Janeiro, 1990. (Dissertação de mestrado).

Von Sperling, M. Introdução à qualidade das águas e ao tratamento de esgotos. 3 . ed. Belo Horizonte: UFMG, Departamento de Engenharia Sanitária, 2005. v. 1. 